Check for updates

Cite this: RSC Adv., 2020, 10, 15282

Received 22nd January 2020

Accepted 9th April 2020

DOI: 10.1039/d0ra00556h

rsc.li/rsc-advances

\section{Silica-immobilized ionic liquid Brønsted acids as highly effective heterogeneous catalysts for the isomerization of $\boldsymbol{n}$-heptane and $\boldsymbol{n}$-octane $\uparrow$}

\begin{abstract}
Abhishek Dhar, $\$^{\mathrm{a}}$ Nadavala Siva Kumar, (D) $\ddagger^{\mathrm{b}}$ Mehul Khimani, $\neq^{\mathrm{c}}$ Ahmed S. Al-Fatesh, ${ }^{\mathrm{b}}$ Ahmed A. Ibrahim, ${ }^{\mathrm{b}}$ Anis H. Fakeeha, ${ }^{\mathrm{b}}$ Hiren Patel ${ }^{\mathrm{c}}$ and Rohit L. Vekariya (D)*de

Metal-free imidazolium-based ionic liquid (IL) Brønsted acids 1-methyl imidazolium hydrogen sulphate $[\mathrm{HMIM}] \mathrm{HSO}_{4}$ and 1-methyl benzimidazolium hydrogen sulphate $\left[\mathrm{HMBIM} \mathrm{HSO}_{4}\right.$ were synthesized. Their physicochemical properties were investigated using spectroscopic and thermal techniques, including UV-Vis, FT-IR, ${ }^{1} \mathrm{H}$ NMR, ${ }^{13} \mathrm{C}-\mathrm{NMR}$, mass spectrometry, and TGA. The ILs were immobilized on mesoporous silica gel and characterized by FT-IR spectroscopy, scanning electron microscopy, Brunauer-Emmett-Teller analysis, ammonia temperature-programmed desorption, and

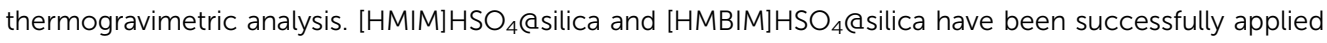
as promising replacements for conventional catalysts for alkane isomerization reactions at room temperature. Isomerization of $n$-heptane and $n$-octane was achieved with both catalysts. In addition to promoting the isomerization of $n$-heptane and $n$-octane (a quintessential reaction for petroleum refineries), these immobilized catalysts are non-hazardous and save energy.
\end{abstract}

\section{Introduction}

Ionic liquids (ILs) are molten salts having melting points below $100{ }^{\circ} \mathrm{C}$. They have received considerable attention over the last decade, due to their numerous inherent features, including low melting temperatures, stability at elevated temperatures and in air, high ionic conductivities, favorable behaviors in solution, large electrochemical windows, low vapor pressures, low volatilities, and non-flammability. ${ }^{\mathbf{1 - 1 0}} \mathrm{ILs}$, also referred to as roomtemperature ILs (RTILs), have been thoroughly investigated as alternatives to conventional catalysts ${ }^{\mathbf{1 1 , 1 2}}$ and are prized for applications in modern green technologies. ${ }^{\mathbf{1 3 , 1 4}}$ They are used in various organic syntheses as solvents or catalysts, such as alkylation of aromatics with olefins, ${ }^{15,16}$ isomerization of light alkanes, ${ }^{17-19}$ and oligomerization of butanes. ${ }^{20-22}$

Homogeneous superacid systems have been shown to catalyze alkane hydroisomerization at temperatures as low as $25^{\circ} \mathrm{C}$.

${ }^{a}$ Department of Chemistry, Modern Institute of Engineering and Technology, Bandel, Hooghly, 712123, West Bengal, India

${ }^{b}$ Department of Chemical Engineering, King Saud University, P.O. Box 800, Riyadh 11421, Saudi Arabia

'School of Sciences, P P Savani University, NH-8, GETCO, Near Biltech, Village: Dhamdod, Kosamba, Dist, Surat 394125, Gujarat, India

${ }^{d}$ Department for Management of Science and Technology Development, Ton Duc Thang University, Ho Chi Minh City, Vietnam.E-mail: rohit.vekariya@tdtu.edu.vn

${ }^{e}$ Faculty of Applied Sciences, Ton Duc Thang University, Ho Chi Minh City, Vietnam $\uparrow$ Electronic supplementary information (ESI) available. See DOI: 10.1039/d0ra00556h

\$ These authors have equally contributed to this work.
These catalysts generally comprise a mixture of strong Lewis acids (e.g., $\left.\mathrm{SbF}_{5}, \mathrm{TaF}_{5}, \mathrm{NbF}_{5}\right)$ and a Brønsted acid $\left(\mathrm{CF}_{3} \mathrm{COOH}\right.$ or $\left.\mathrm{CF}_{3} \mathrm{SO}_{3} \mathrm{H}\right) .{ }^{23}$ However, free $\mathrm{HF}$ in these homogeneous superacids makes them highly toxic and corrosive. The two major obstacles to the application of ILs remain cost and availability. Although a wide range of IL synthetic approaches have been published, most of these methods are complex and require extensive purification after synthesis in order to afford pure, dry RTILs. In addition, the high cost of some ILs limits their commercial availability and application. Thus, isomerization by ILs remains a topic of considerable interest worldwide. ${ }^{\mathbf{2 4 - 2 6}}$

Global investigations have led to the concept of immobilized ILs. ${ }^{14,18}$ For example, ILs have been anchored to the exterior facets and cavities of various porous solid materials to make ILs more cost-efficient. The thin IL layer allows for faster diffusion and mass transfer, due to the higher relative viscosities of many RTILs. Catalysts are physisorbed on silica gel to improve IL yields, simplify purification, lengthen catalyst longevity, reduce exposure to hazardous chemicals, and increase recyclability. Applications of such catalysts are a worldwide priority.

Acids having a silica gel matrix, for example silicate polyphosphoric acid and silicate $\mathrm{HClO}_{4}$, have been investigated for organic fabrication due to their inherent characteristics, such as high performance, high thermal stability and recyclability, low toxicity, increased selectivity, and convenience. ${ }^{27-29}$ In recent reports, fluoroboric acid (a weak protonic acid) was uptake on silica to prevent unwanted side reactions. ${ }^{30}$ Silicate sulfuric acid has been studied as a catalyst in two different ways: as silicaadsorbed sulfuric acid and as silica sulfuric acid. The 


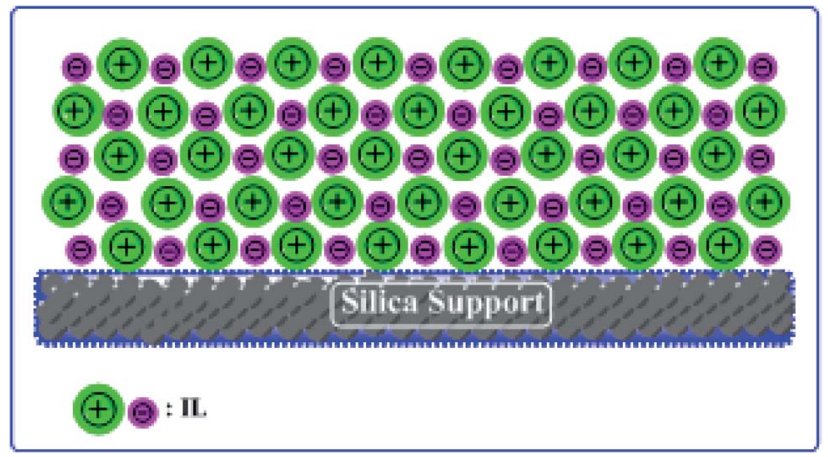

Scheme 1 Schematic illustration of supported IL catalyst.

fabrication of silica-adsorbed sulfuric acid is fairly simple and inexpensive for huge scale production, since it can be simply reused without altering the activity of the catalytic system. It is therefore considered an environmentally friendly and reusable catalyst. ${ }^{31}$ Silica-adsorbed sulfuric acid is a strong alternative to sulfuric acid or chlorosulfonic acid, despite some limitations, for instance the loss of acidophilic functional groups, employment of toxic solvents, and requirement of costly reagents or solvents. ${ }^{32}$ At the same time, silica-sulfuric acid is an alternative catalyst for some specific chemical reactions to increase yield and improve other factors. In addition to silica, other solid support media have been used and numerous reports of polymer matrixes have been published. However, silica remains the preferred catalyst support.

Herein, we report two different ILs and silica-supported catalysts. We have chosen silica gel as a support for the ILs and have prepared heterogeneous catalysts with good acidity and thermal stability (Scheme 1).

\section{Materials}

\subsection{Chemicals}

$N$-Methylimidazole, $N$-methylbenzimidazole, and silica gel Davisil@ grade 633 were attained from Sigma-Aldrich. HCl,

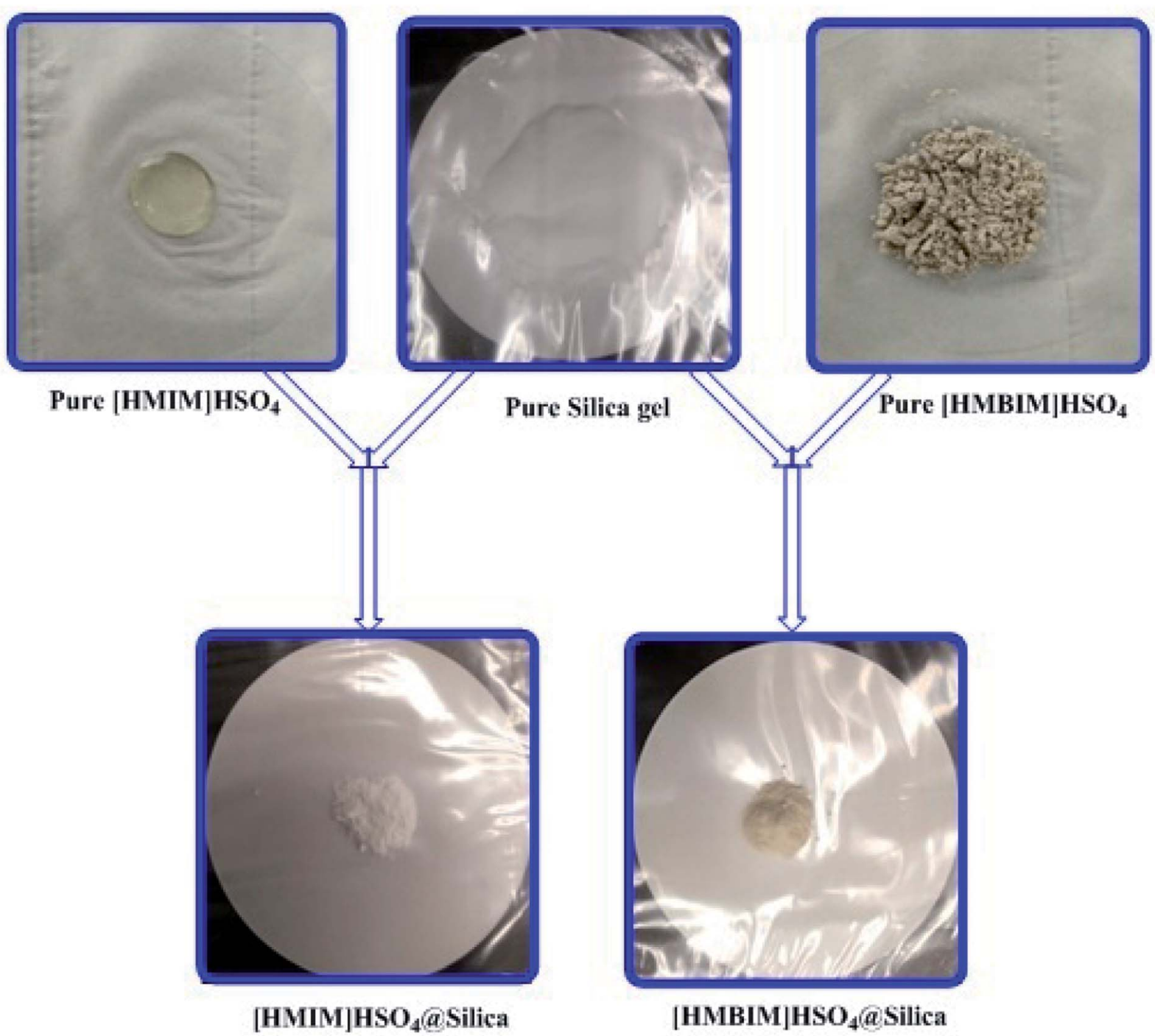

Fig. 1 Physical appearances of silica supported IL catalysts. 


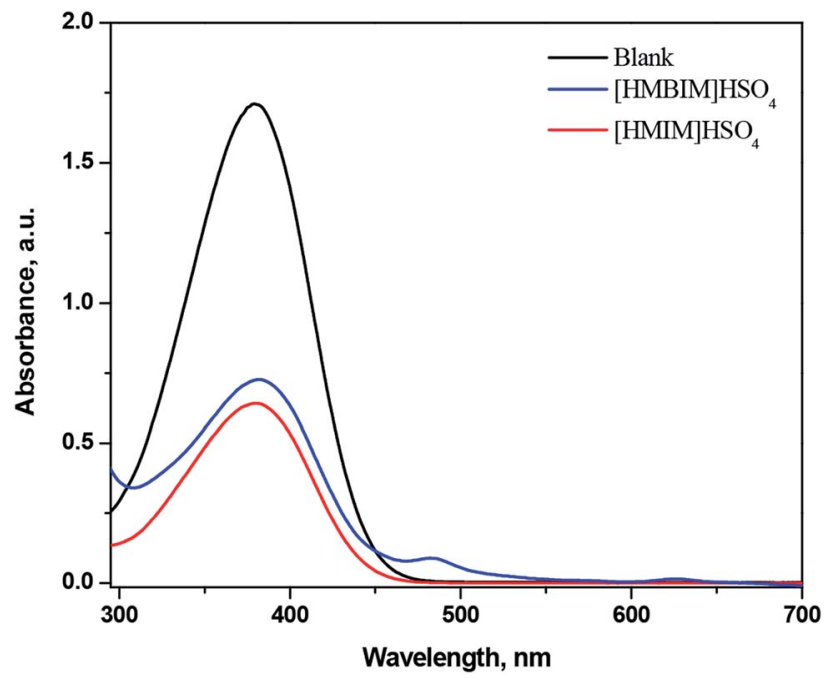

Fig. 2 UV-Vis spectra of 4-nitroaniline after adding ILBAs in water.

$\mathrm{H}_{2} \mathrm{SO}_{4}$ and other AR grade reagents were purchased and used without additional purification.

\subsection{Methods}

The vibrational modes of prepared samples were recorded in the range $4000-500 \mathrm{~cm}^{-1}$ with a FTIR Spectrometer (IR Prestige21, SHIMADZU). ${ }^{1} \mathrm{H}$ and ${ }^{13} \mathrm{C}$ NMR spectra were registered using a NMR spectrometer (Bruker AVANCE III $400 \mathrm{MHz}$ ) with TMS as the internal standard and $\mathrm{D}_{2} \mathrm{O}$ as the solvent. TG-DTA were performed via a Setsys Evolution TGA-DTA/DSC, with nitrogen flow and temperature ramped at $5{ }^{\circ} \mathrm{C} \mathrm{min}^{-1}$, from $25-800{ }^{\circ} \mathrm{C}$. The mass spectra were recorded with "Thermo Fisher Exactive Plus High Resolution Mass Spectrometer". Sample shapes and surface morphologies were obtained using SEM (Hitachi S-4800, Japan). Nitrogen adsorption-desorption isotherms were captured at $-196{ }^{\circ} \mathrm{C}$ using a 3 Flex Micrometric (US) sorption surface unit. All samples were degassed at $130^{\circ} \mathrm{C}$ for $12 \mathrm{~h}$ prior to reading. The total surface area has been calculated according to Brunauer-Emmett-Teller (BET) model by using software supplied with the apparatus. Total pore volumes were measured at $P / P_{0}=0.95$, supposing the entire surface to be saturated with $\mathrm{N}_{2}$. The Barrett-Joyner-Halenda (BJH) model was used to calculate pore size distributions. Ammonia temperatureprogrammed desorption ( $\left.\mathrm{NH}_{3}-\mathrm{TPD}\right)$ measurements were performed on an Autochem 2920 Micrometric (US) to measure the acidity of silica-supported ILs; ammonia was used as the adsorbate. Approximately $0.2 \mathrm{~g}$ of sample was used in a quartz reactor and saturated with ammonia at $25^{\circ} \mathrm{C}$. Next, the samples were purged with argon to eliminate residual $\mathrm{NH}_{3}$ from the upperlayer of the samples. TPD was performed from 100 to $800{ }^{\circ} \mathrm{C}$ with a heating rate of $10{ }^{\circ} \mathrm{C} \mathrm{min}^{-1}$ using argon $(30$ $\mathrm{mL} \min ^{-1}$ ) as the carrier gas.

\subsection{Synthesis of [HMIM] $\mathrm{HSO}_{4}$ and [HMBIM] $\mathrm{HSO}_{4}$}

To begin, 1-methyl imidazole ( $5.0 \mathrm{~g}, 0.060 \mathrm{~mol}), 1$-methyl benzimidazole $(5.0 \mathrm{~g}, 0.037 \mathrm{~mol})$, and acetonitrile $(10 \mathrm{~mL})$ were mixed
Table 1 Computation and assessment of $H_{0}$ parameters of BAILs in $\mathrm{H}_{2} \mathrm{O}$ at $30{ }^{\circ} \mathrm{C}$ by using 4 -nitroaniline indicator

\begin{tabular}{llrrll}
\hline Entry & ILs & $A_{\max }$ & {$[\mathrm{I}](\%)$} & {$\left[\mathrm{IH}^{+}\right](\%)$} & $H_{0}$ \\
\hline 1 & No IL & 1.708 & 100.0 & 0 & - \\
2 & {$[\mathrm{HMIM}] \mathrm{HSO}_{4}$} & 0.641 & 37.5 & 62.5 & 0.76 \\
3 & {$[\mathrm{HMBIM}] \mathrm{HSO}_{4}$} & 0.726 & 42.5 & 57.5 & 0.85
\end{tabular}

in a round-bottom flask with stirring for 5 min at $0{ }^{\circ} \mathrm{C}$. Next, a fixed volume of concentrated $\mathrm{H}_{2} \mathrm{SO}_{4}$ was added dropwise via an adjusting funnel and the mixture was agitated for $1 \mathrm{~h}$ at $0{ }^{\circ} \mathrm{C}$. Subsequently, the stirring was continued at $25{ }^{\circ} \mathrm{C}$ for an additional $1 \mathrm{~h}$. The synthesized ILs were washed with $\left(\mathrm{C}_{2} \mathrm{H}_{5}\right)_{2} \mathrm{O}$ to eliminate any nonionic residues and vacuum-dried at $80{ }^{\circ} \mathrm{C}$ for $24 \mathrm{~h}$. Finally, viscous or oily ([HMIM] $\mathrm{HSO}_{4}$ ) and solid ([HMBIM] $\mathrm{HSO}_{4}$ ) were stored in sealed tubes under inert argon atmosphere. (Characterizations have been given in ESI: Fig. S1 and S2 †).

\subsection{UV-Vis acidity evaluation}

Prior to use, ILs were dehydrated under vacuum for 2 hours at $80^{\circ} \mathrm{C}$. De-ionized water were taken in the solution preparations. UV-Vis spectra were gathered by means of spectrophotometer "Agilent B453".

\subsection{IL@silica preparation}

Silica gel Davisil $®$ grade 633 (average pore diameter $6 \mathrm{~nm}$, pore volume $0.75 \mathrm{~cm}^{3} \mathrm{~g}^{-1}, 200-425$ mesh particle size) was first refluxed with $6 \mathrm{M} \mathrm{HCl}$ for $1440 \mathrm{~min}$, then splashed with double distilled $\mathrm{H}_{2} \mathrm{O}$ to maintain the solution $\mathrm{pH}$ between 6 to 7 , after that dried overnight at $100{ }^{\circ} \mathrm{C}$. The combination of activated silica gel $(5.0 \mathrm{~g})$ and $30 \mathrm{~mL} \mathrm{C}_{2} \mathrm{H}_{5} \mathrm{OH}$ was added each to two $0.1 \mathrm{~L}$ three-necked round-bottom flasks. Next, a solution of $4.0 \mathrm{~g}$ of

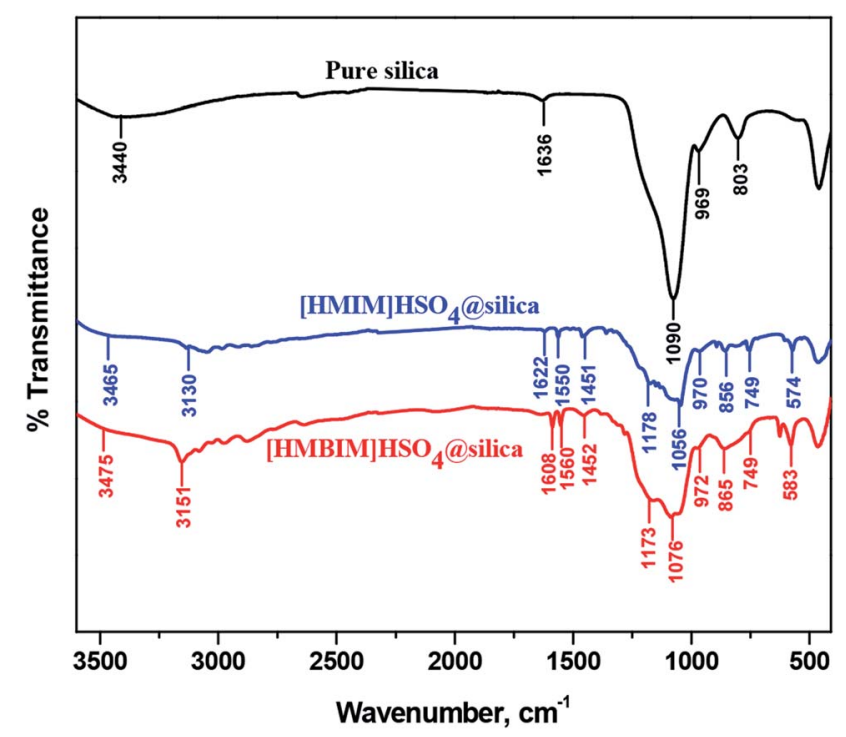

Fig. 3 Comparison of FT-IR spectra of pure silica, [HMIM] $\mathrm{HSO}_{4}$ @asilica (CAT-1), and [HMBIM] $\mathrm{HSO}_{4}$ (asilica (CAT-2). 
[HMIM] $\mathrm{HSO}_{4}$ and a solution of $4.0 \mathrm{~g}$ of $[\mathrm{HMBIM}] \mathrm{HSO}_{4}$ in $20 \mathrm{~mL}$ of $\mathrm{C}_{2} \mathrm{H}_{5} \mathrm{OH}$ were dropped slowly to their respective roundbottom flasks under nitrogen atmosphere. Subsequently $180 \mathrm{~min}$ of stirring at $25^{\circ} \mathrm{C}$, the solvent was evaporated under low pressure to get the IL@silica as a white/light brown powder (40 wt\% [HMIM] $\mathrm{HSO}_{4} /$ silica gel and [HMBIM] $\mathrm{HSO}_{4} /$ silica gel). The physical appearances of pure ILs and ILs@silica gel are shown in Fig. 1.

\subsection{Catalytic performance assessment}

Catalytic performance was measured in a $30 \mathrm{~mL}$ stainless steel autoclave. A calculated amount of the feed stock ( $n$-heptane and $n$-octane) and the fabricated IL catalyst were added to the autoclave, which was partially dipped in a fixed temperature oil bath, and the mixture was agitated at $1500 \mathrm{rpm}$. The pressure inside the autoclave was maintained at approximately $1 \mathrm{MPa}$ and the reaction time was $12 \mathrm{~h}$. Next the completion of the reaction, the autoclave was cooled to $25^{\circ} \mathrm{C}$. The reaction system separated into two phases immediately. Hence, contact time (reaction time) was considered as the time from introduction of the feedstock to cessation of stirring. The isomerized products were evaluated by gas chromatography "GC, Shanghai Haixin Chromatographic Instrument Co. Ltd., Model: GC-950FID, Column: CP-7531" after collecting liquid samples via syringe. The tests were performed in batch mode in the range of 20$40{ }^{\circ} \mathrm{C}$. The $\mathrm{IL} / n$-alkane volume ratio was fixed at optimized $0.5: 1 .^{18}$

\section{Results and discussion}

\subsection{Determination of $\boldsymbol{H}_{0}$ values for IL Brønsted acids}

UV-visible spectrophotometry was performed using an alkaline indicator to measure the Brønsted acidic strength of ILs. ${ }^{33,34}$ The
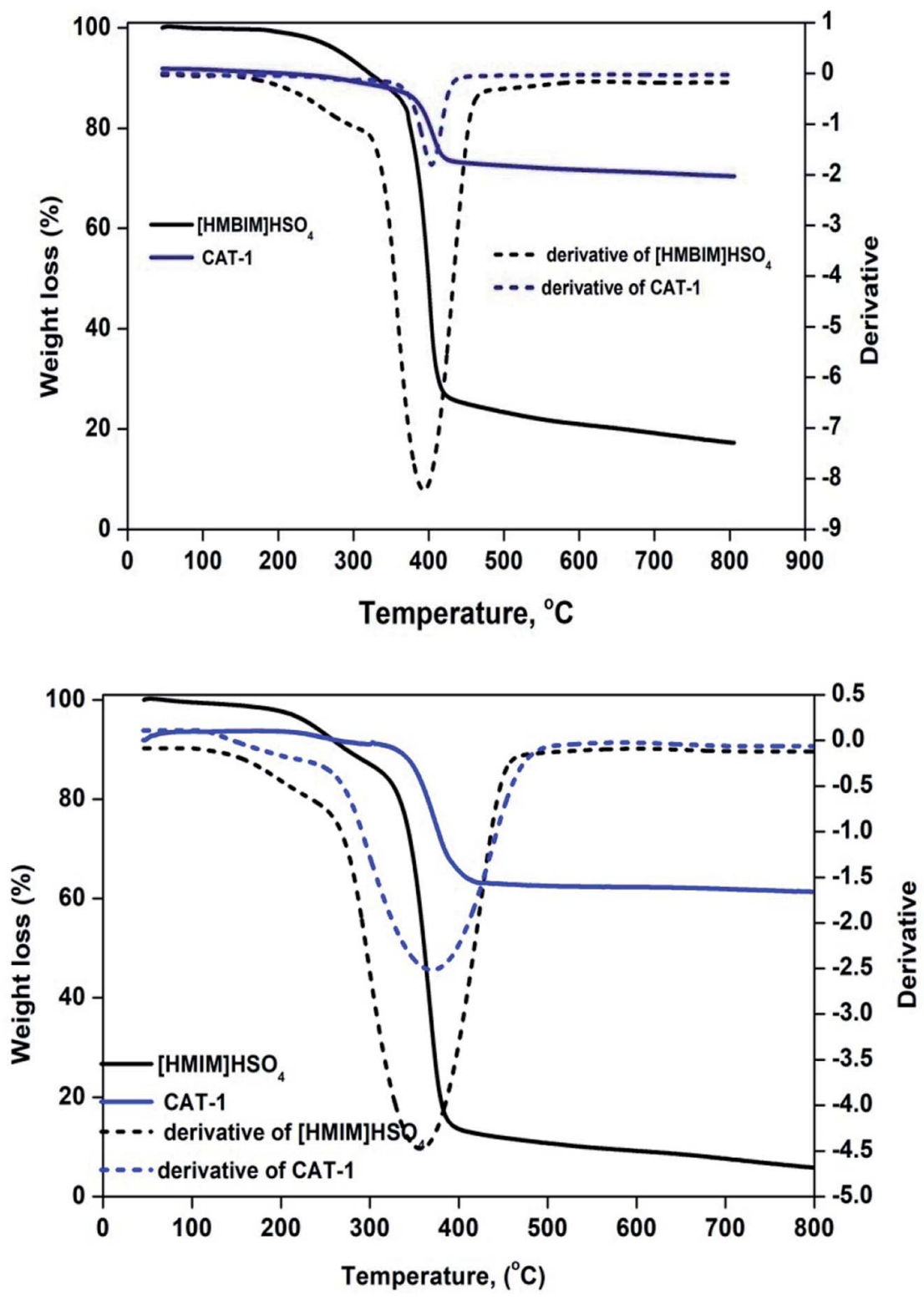

Fig. 4 TGA curves for pure ILs and IL@asilica. 
absorbance of the non-protonated basic indicator decreases with increasing acidity of the IL. The protonated state of the indicator has a small molar absorptivity, thus $[\mathrm{I}] /\left[\mathrm{IH}^{+}\right]$(where I denotes the indicator) can be calculated from the difference in absorbance measured after adding the IL Brønsted acids (ILBAs). Eqn (1) was employed to determine the Hammett function, $H_{0}$. This value describes the comparative acidity of the ILs;

$$
H_{0}=\mathrm{p} K(\mathrm{I})_{\mathrm{aq}}+\log \left(\frac{[\mathrm{I}]}{\left[\mathrm{IH}^{+}\right]}\right)
$$

Using $10 \mathrm{mg} \mathrm{L}^{-1}$ of 4-nitroaniline $\left(\mathrm{p} K(\mathrm{I})_{\mathrm{aq}}=0.99\right)$ and $25 \mathrm{mmol} \mathrm{L}^{-1}$ IL in dichloromethane, the $H_{0}$ values for IL BAs were computed. The form of non-protonated indicator displayed a maximum absorbance at approximately $378 \mathrm{~nm}$ in water. The absorbance of the non-protonated form of the indicator diminished adding acidic IL to the solution. Fig. 2 demonstrates that the absorbance of the non-protonated form of the indicator for the two acidic ILs comes in the order: $[\mathrm{HMBIM}] \mathrm{HSO}_{4}>[\mathrm{HMIM}] \mathrm{HSO}_{4}$.

The order of acidity of the two ILs was determined with the subsequent $H_{0}$ values (Table 1): [HMIM] $\mathrm{HSO}_{4}(0.76)>$ [HMBIM] $\mathrm{HSO}_{4}$ (0.85), which confirms that $[\mathrm{HMIM}] \mathrm{HSO}_{4}$ is a stronger Brønsted acid than [HMBIM] $\mathrm{HSO}_{4}$. It is evident from the structures of the two synthesized ILs that the $[\mathrm{HMBIM}] \mathrm{HSO}_{4} \mathrm{IL}$ provides increased steric hindrance to the acidic proton residing on $\mathrm{N}^{+}$, making generation of a 'naked' proton is less favorable. As a result, the Brønsted acidity of $[\mathrm{HMBIM}] \mathrm{HSO}_{4} \mathrm{IL}$ is reduced. The acidic strength of the ILs depends on the features of both the cations and anions. When the cations of the ILs were the same, the acidity of the IL was controlled by the anion type.

\subsection{Characterization of catalysts}

3.2.1 FT-IR. The synthesized catalysts were also characterized using FTIR. The spectra of silica alone and the silicasupported ILs, [HMIM] $\mathrm{HSO}_{4} @$ silica (CAT-1) and [HMBIM] $\mathrm{HSO}_{4} @$ silica (CAT-2), are shown in Fig. 3.

FT-IR analysis confirms the successful preparation of both the CAT-1 and CAT-2 catalysts. Characteristic spectral bands were observed for CAT-1 and CAT-2 at $749 \mathrm{~cm}^{-1}$ (attributed for C-H stretching), 865 and $856 \mathrm{~cm}^{-1}$ (C-H bending), $\sim 1050 \mathrm{~cm}^{-1}$ ( $\mathrm{S}=\mathrm{O}$ stretching), 1173 and $1178 \mathrm{~cm}^{-1}$ (C-N stretching), 1451 and $1452 \mathrm{~cm}^{-1}$ (C-N band of the imidazolium ring of the supported ILs). Silica support increases the effective surface area of the catalysts, which in turn enhances the isomerization reaction yields. In addition, the large covalently bonded silica network increases the thermodynamic stability of the catalysts.

3.2.2 TGA. ILs are considered to be thermally stable, due to their extremely high decomposition temperatures. Thermal stability measurements were performed by thermogravimetric analysis (TGA) as a function of \% weight loss vs. temperature $\left({ }^{\circ} \mathrm{C}\right)$. Fig. 4 displays TGA curves for CAT- 1 and CAT-2. Decomposition temperatures $\left(T_{\mathrm{d}}\right)$ can be defined by several definitions, including start, \% mass loss, and peak $\left(T_{\text {peak }}\right)$ temperatures. Weight loss from both pure ILs and silica-supported IL catalysts at temperatures below $100{ }^{\circ} \mathrm{C}$ is ascribed to desorption of left

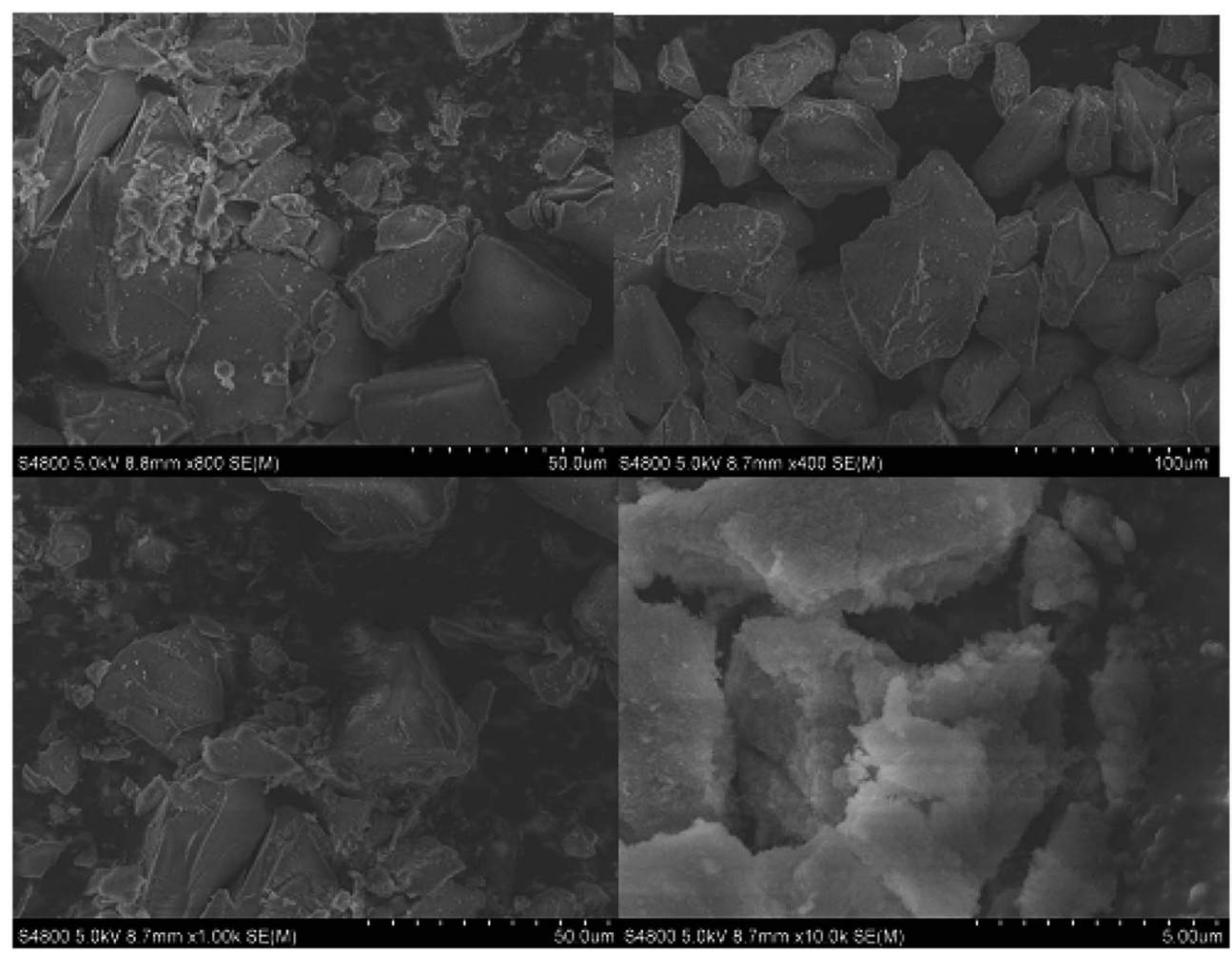

Fig. 5 SEM images of pure silica gel (without immobilized IL). 


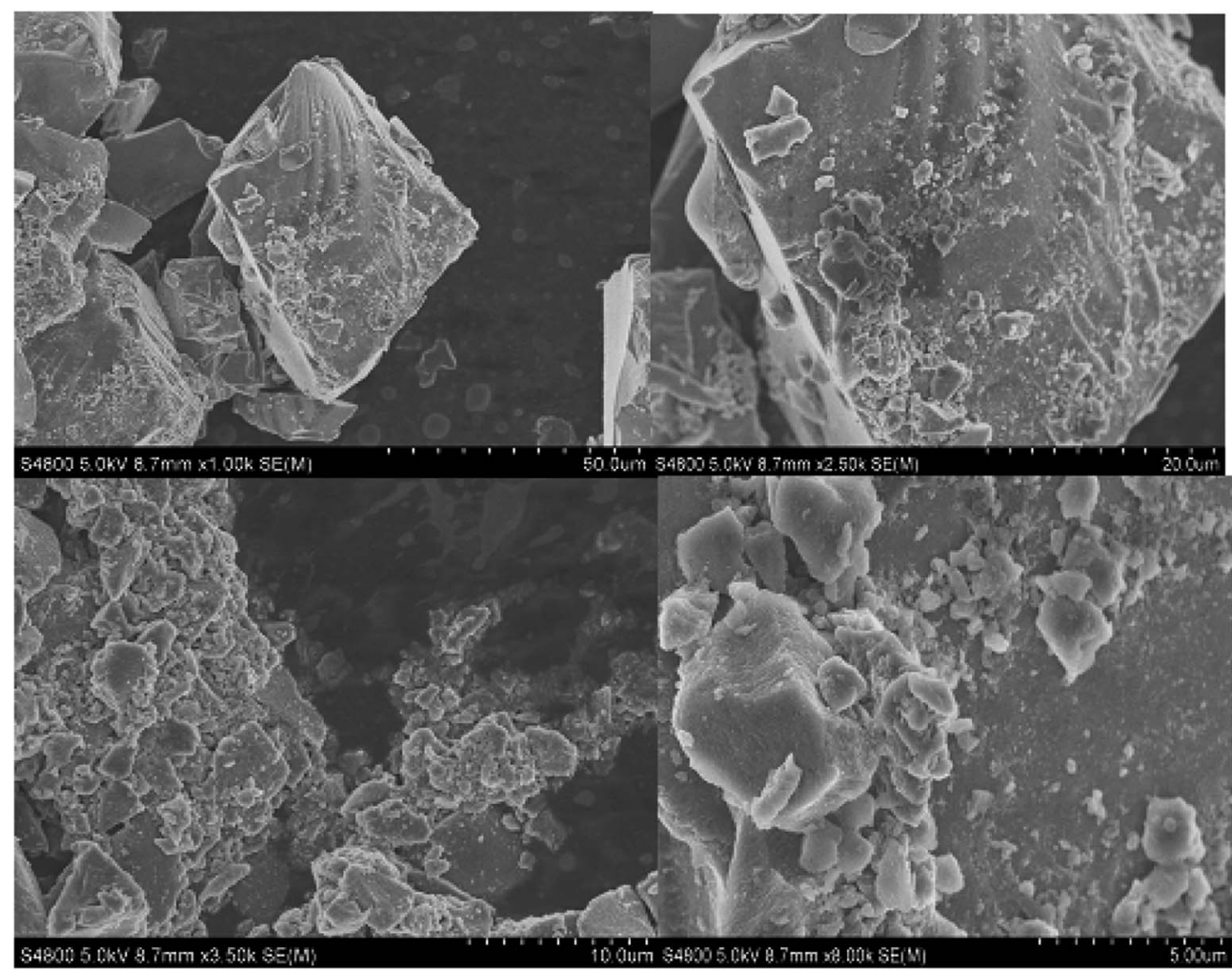

Fig. 6 SEM images of CAT-1 (silica gel with immobilized IL (HMIM-HSO 4$)$ ).
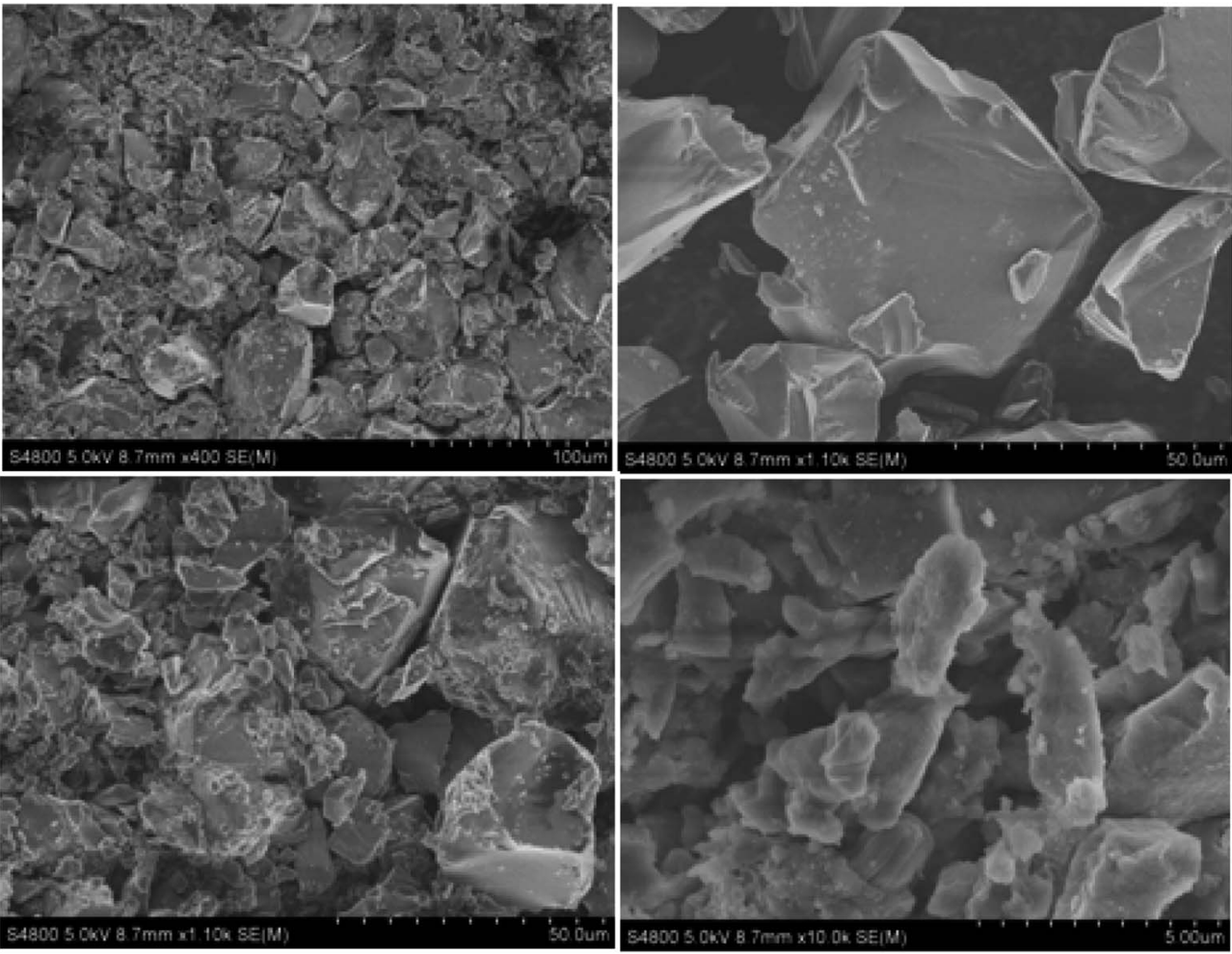

Fig. 7 SEM images of CAT-2 (silica gel with immobilized IL $\left(\mathrm{HMBIM}-\mathrm{HSO}_{4}\right)$ ). 

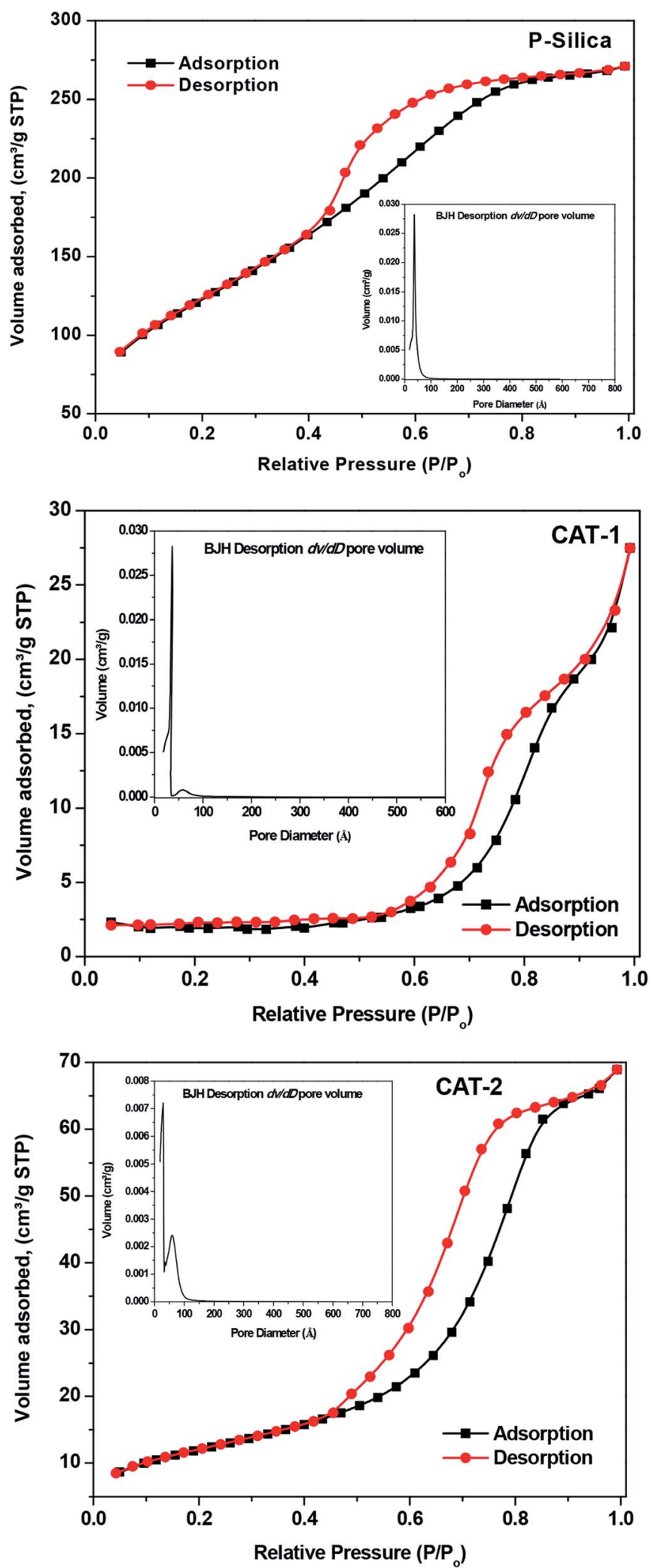

Fig. $8 \mathrm{~N}_{2}$ adsorption-desorption isotherms and the corresponding $\mathrm{BJH}$ desorption $\mathrm{d} V / \mathrm{d} D$ pore volume derived from the adsorption isotherm (inset) of pure silica and silica-supported ILs. over physisorbed $\mathrm{H}_{2} \mathrm{O}$ and/or organic solvent molecules from pore channels used at the beginning of catalyst preparation. The temperature at which the sample begins to lose mass is known as $T_{\text {start }}{ }^{35}$ For both ILs, $T_{\text {start }}$ is approximately $110{ }^{\circ} \mathrm{C}$. However, both supported catalysts are thermally stable up to at least $341{ }^{\circ} \mathrm{C}$ (CAT-1) and $381{ }^{\circ} \mathrm{C}$ (CAT-2). Degradation of $10 \%$ $[\mathrm{HMIM}] \mathrm{HSO}_{4},[\mathrm{HMBIM}] \mathrm{HSO}_{4}, \mathrm{CTA}-1$, and CTA-2 occurs at $273.1,329.6,359.8$, and $395.4{ }^{\circ} \mathrm{C}$, respectively.

The first derivative of the weight loss curve can be utilized to determine the point at which the most explicit weight loss occurs. The first derivative peak temperatures for [HMIM] $\mathrm{HSO}_{4}$, [HMBIM] $] \mathrm{HSO}_{4}$, CTA-1, and CTA-2 are 359, 391, 365.1, and $402.5{ }^{\circ} \mathrm{C}$, respectively. For imidazolium-based ILs, degradation occurs through a variety of mechanisms, the most common of which is loss of major alkyl chains. ${ }^{36,37}$ In our case, both ILs also follow this mechanism. Increasing temperature leads to cleavage of the methyl group; these results also correlate with mass spectral evidence. However, benzene substitution provides more thermal stability to the IL. The maximum degradation temperatures for [HMIM] $\mathrm{HSO}_{4}$ and [HMBIM] $\mathrm{HSO}_{4}$ are 384.7 and $413.9{ }^{\circ} \mathrm{C}$, respectively. Beyond that temperature, no significance weight loss is observed up to $800{ }^{\circ} \mathrm{C}$. The maximum weight losses were approximately $27.01 \%$ and $12.92 \%$ over temperature ranges of $381.2-423.5{ }^{\circ} \mathrm{C}$ and $341.7-$ $417.7{ }^{\circ} \mathrm{C}$ for CAT-1 and CAT-2, respectively. Slow degradation at higher temperatures may attributed to the larger number of $\mathrm{Si}-$ $\mathrm{O}-\mathrm{Si}$ bonds formed in imidazolium IL, which could hinder thermal decomposition significantly. However, at extremely high temperatures, the covalently attached ILs decomposed (dissociation of imidazolium moieties) from the top layer of the silica gel. Hence, the final observed weight losses occurred from 417.0-790.7 ${ }^{\circ} \mathrm{C}(\sim 2.31 \%)$ for CAT-1 and $423.5-790.0{ }^{\circ} \mathrm{C}$ ( $3.54 \%)$ for CAT-2.

3.2.3 Morphological studies by scanning electron microscopy. SEM was used to investigate the geometry and surface properties of pure silica and silica-supported IL samples (Fig. 57). There is no significance difference in particle size between the silica gel and the silica-supported ILs. This indicates appreciable mechanical stability of the silica gel particles during immobilization. Nevertheless, the surface morphologies of the two samples are quite different. Fig. 5 demonstrates that the $\mathrm{SiO}_{2}$ surface is thin and that small agglomerates are found on the top layer of silica-supported IL (Fig. 6 and 7). No apparent changes in crystal morphology were observed when ILs were loaded onto silica at $40 \%$, as shown in the SEM micrographs. Morphology changes are not expected to occur during sintering of the IL species, otherwise it would not be feasible to recycle the catalyst. Hence, we conclude that the silica crystals remain intact after the reversible immobilization of catalyst particle agglomerates.

3.2.4 $\mathrm{N}_{2}$ adsorption-desorption determination of catalyst pore structures and surface areas. $\mathrm{N}_{2}$ adsorption-desorption experiments were used to estimate the surface aspects of CAT-1 and CAT-2 using the BET and BJH methods. The samples exhibit type-IV isotherms, confirming the mesoporous nature of the synthesized IL@silica catalysts, as well as that of silica (Fig. 8). This type of isotherm confirms capillary condensation 
Table 2 Results of $\mathrm{N}_{2}$ adsorption measurements of the pure silica gel (P-silica) and ILs supported silica (IL@silica)

\begin{tabular}{llll}
\hline Sample & $\begin{array}{l}\text { BET surface } \\
\text { area }\left[\mathrm{m}^{2} \mathrm{~g}^{-1}\right]\end{array}$ & $\begin{array}{l}\text { Single desorption } \\
\text { pore volume }\left[\mathrm{cm}^{3} \mathrm{~g}^{-1}\right]\end{array}$ & $\begin{array}{l}\text { BJH desorption } \\
\text { average pore diameter }[\AA]\end{array}$ \\
\hline Pure silica gel & 445.6 & 0.415 & 36.14 \\
CAT-1 ([HMIM] & 5.45 & 0.033 & 85.97 \\
CAT-2 ([HMBIM $] \mathrm{HSO}_{4} @$ @ilica gel) & 43.14 & 0.101 & 60.0
\end{tabular}
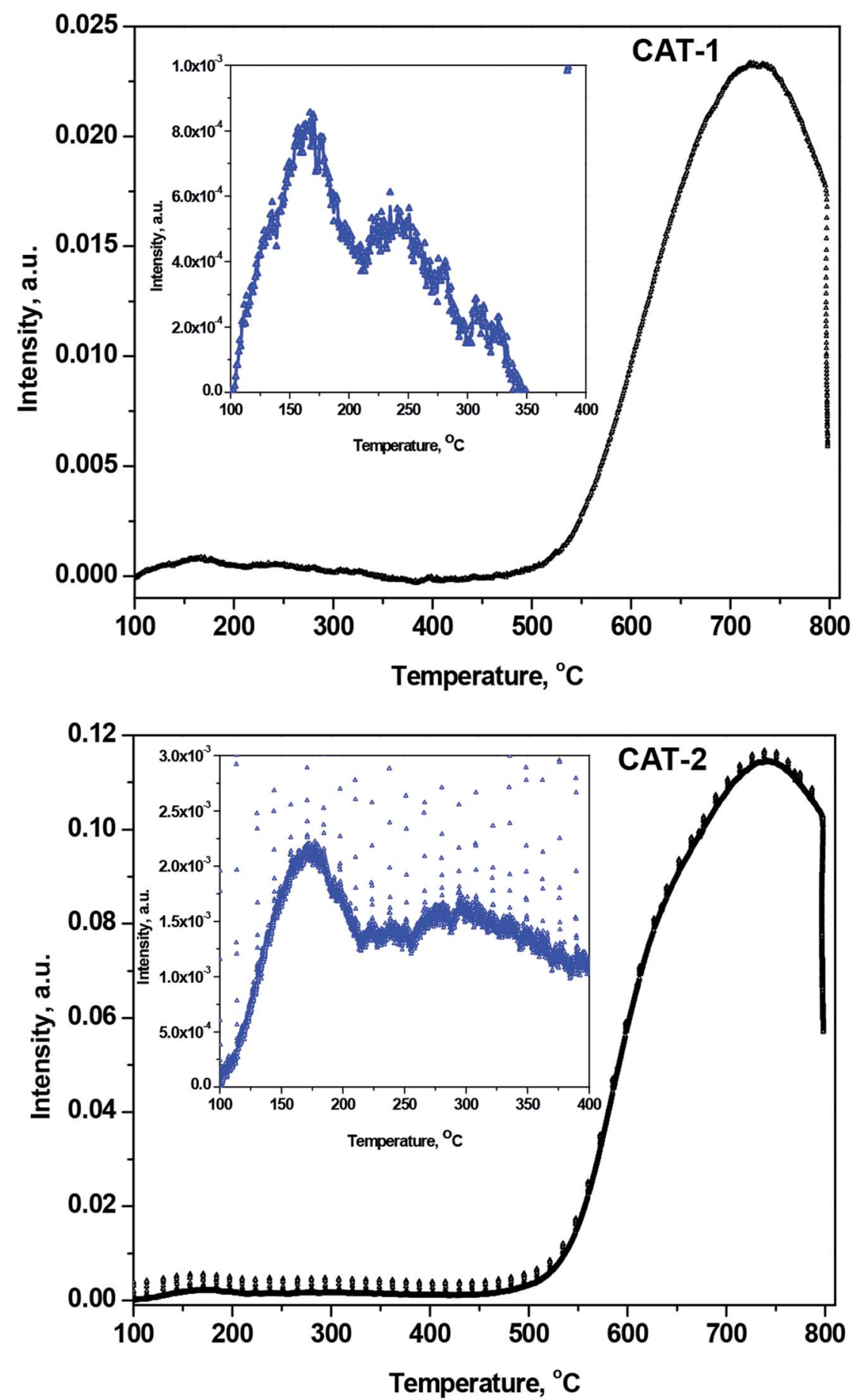

Fig. $9 \mathrm{NH}_{3}$-TPD curves for desorbed $\mathrm{NH}_{3}$ after heating from 100 to $800{ }^{\circ} \mathrm{C}$ for CAT-1 and CAT-2. Inset curve is the magnification of lower temperature range scale for clarity. 
during the adsorption process. "Ink-bottle" type pores are confirmed by the presence of $\mathrm{H} 2$ hysteresis loops having adsorption and desorption branches at comparative pressures of 0.8 and 0.4 , respectively. ${ }^{38}$ Both catalysts have lower surface pore volumes and surface areas compared to pure silica gel. However, the specific surface area decreases in most cases after the introduction of the IL, due to micro- or mesopore blocking. ${ }^{39}$ Interestingly, comparison of the BET results for silicasupported ILs and pure silica indicates that the surface area of silica-supported ILs is less than that of pure silica. The average pore diameter and surface area of pure silica mesoporous material are $36.14 \AA$ and $445.6 \mathrm{~m}^{2} \mathrm{~g}^{-1}$, respectively. Upon mixing with $\mathrm{IL}$, the pore diameters increase to $85.97 \AA$ (CAT-1) and 60.0 $\AA$ (CAT-2) and surface areas decrease to 5.45 $\mathrm{m}^{2} \mathrm{~g}^{-1}$ (CAT-1) and $43.14 \mathrm{~m}^{2} \mathrm{~g}^{-1}$ (CAT-2). Subsequently, the pore volume of 0.415 for pure silica decreases to 0.033 and 0.101 for CAT-1 and CAT-2, respectively (Table 2). The attachment of bulky imidazolium or benzimidazolium cations to the framework, which increases strain on the meso structured, is also responsible for the increased activity of the catalysts, ${ }^{\mathbf{4 0 , 4 1}}$ since alkane molecules are more exposed at the surface, facilitating the isomerization process.

Furthermore, as shown in the inset of Fig. 8, pore-size distribution was quite narrow for pure silica and widened with a new peak arising on the higher diameter side when $40 \mathrm{wt} \%$ IL was immobilized on silica. This indicates that ILs are perhaps confined to the silica gel poresat these concentrations.

3.2.5 $\mathrm{NH}_{3}$-TPD acidity measurements. The band in the TPD curves can be segmented into different well-defined component bands having various maxima over the temperature range 100$800{ }^{\circ} \mathrm{C}$ (Fig. 9). The component bands indicate physisorbed, proton-held, and acid-bound $\mathrm{NH}_{3}$. Among the desorbed $\mathrm{NH}_{3}$ molecules, those appearing at $\leq 150{ }^{\circ} \mathrm{C}$ correspond to physically adsorbed and proton-bound $\mathrm{NH}_{3},{ }^{\mathbf{4 2 , 4 3}}$ while the desorbed $\mathrm{NH}_{3}$ molecules observed at higher temperatures correspond to acidbound $\mathrm{NH}_{3}$. The amount of physisorbed and proton-bound $\mathrm{NH}_{3}$ desorbed at up to $\sim 150{ }^{\circ} \mathrm{C}$ declined with increasing temperatures, as the interlayer space of silica gel decreased after immobilization with IL.
The increased acidity of silica-supported ILs results in a greater desorption temperature for $\mathrm{NH}_{3}$ uptake on the acid sites. $^{\mathbf{4 4}}$ The acidity of silica-supported ILs increases due to the presence of Lewis acidic $\mathrm{Si}^{4+}$ centers in silica. The acid sites are defined as weak, medium, strong, and very strong, corresponding to desorption temperatures of 150-250, 250-350, 350500 , and $>500{ }^{\circ} \mathrm{C}$, respectively. Fig. 9 clearly demonstrates that only the weak, medium (inset of Fig. 9), and very strong acid sites exist in silica@IL. After heating, the number of weak acid sites decreases, and the number of medium and very strong acid sites increases.

\subsection{Catalytic activities}

Isomerization of $n$-hexane and $n$-octane in the presence of CTA1 and CTA-2 ILs were studied with a contact time of $12 \mathrm{~h}$ as a function of temperature. The obtained results are displayed in Table 1. It is clear from Tables 3 and 4 that both catalysts isomerize $n$-heptane and $n$-octane to an appreciable extent. Dzhikiya et al. ${ }^{45}$ evaluated the isomerization of $n$-hexane using $\mathrm{Pd} / \mathrm{SO}_{4} / \mathrm{ZrO}_{2} / \mathrm{Al}_{2} \mathrm{O}_{3}$ catalysts and determined that conversion was independent of reaction temperature. However, the yield of isomers decreased with increasing temperature. In contrast, our experimental data demonstrates that the isomerization $n$ hexane and $n$-octane to their respective isomers (2,2-dimethylpentane, 2,3-dimethylpentane, 3-ethylpentane, and 3methyl-1-hexene) increases with temperature from 20 to $30{ }^{\circ} \mathrm{C}$. This is due to enhanced cracking and disproportionation reactions. At high reaction temperatures, higher isomer yields are thermodynamically favorable. However, conversion of $n$ hexane decreases with a temperature increase of $20^{\circ} \mathrm{C}$, since cracking and disproportionation reactions dominate at higher temperatures. In contrast, at $40{ }^{\circ} \mathrm{C}$, yields were approximately equal; these equilibrium yields are easily achieved at high temperatures due to high reaction rates (kinetic limitation). Ibragimov et al. ${ }^{46}$ observed similar results for the isomerization of $n$-hexane in 1-methyl-3-butylimidazolium chloride with $\mathrm{AlCl}_{3}$.

Our synthesized catalysts achieved better isomerization yields than previously reported IL catalysts, such as super acidic

Table 3 Percentage conversion of $n$-heptane and $n$-octane to their corresponding isomers by CAT- 1 after $12 \mathrm{~h}$ at different temperatures

$\%$ of isomers

Temperature $\left({ }^{\circ} \mathrm{C}\right)$

\begin{tabular}{|c|c|c|c|c|}
\hline Starting alkane & Name of isomers & 20 & 30 & 40 \\
\hline & 2,3-Dimethyl pentane & $45.1( \pm 1.5)$ & $46.9( \pm 0.1)$ & $46.2( \pm 0.2)$ \\
\hline & 3-Methyl-1-hexene & $0.27( \pm 0.01)$ & $0.28( \pm 0.02)$ & $0.29( \pm 0.02)$ \\
\hline & $n$-Heptane & $19.43( \pm 0.3)$ & $8.52( \pm 0.2)$ & $9.71( \pm 0.3)$ \\
\hline \multirow[t]{4}{*}{$n$-Octane } & 3-Methyl heptanes & $19.1( \pm 1.3)$ & $21.2( \pm 1.1)$ & $14.8( \pm 0.9)$ \\
\hline & 2,3-Dimethyl hexane & $3.1( \pm 0.2)$ & $18.6( \pm 0.01)$ & $12.4 .( \pm 1.2)$ \\
\hline & 2,3-Dimethyl-2-hexene & $0.35( \pm 0.03)$ & $0.39( \pm 0.04)$ & $0.39( \pm 0.04)$ \\
\hline & $n$-Octane & $52.25( \pm 0.6)$ & $37.21( \pm 1.1)$ & $36.61( \pm 0.2)$ \\
\hline
\end{tabular}


Table 4 Percentage conversion of $n$-heptane and $n$-octane to their corresponding isomers by CAT- 2 after $12 \mathrm{~h}$ at different temperatures

\begin{tabular}{|c|c|c|c|c|}
\hline \multirow{2}{*}{ Starting alkane } & \multirow{2}{*}{ Name of isomers } & \multicolumn{3}{|l|}{$\%$ of isomers } \\
\hline & & \multicolumn{3}{|c|}{ Temperature $\left({ }^{\circ} \mathrm{C}\right)$} \\
\hline \multirow[t]{3}{*}{$n$-Heptane } & 2,2-Dimethyl pentane & $16.2( \pm 0.1)$ & $19.3( \pm 0.2)$ & $17.4( \pm 0.3)$ \\
\hline & 2,3-Dimethyl pentane & $42.3( \pm 1.2)$ & $43.1( \pm 0.3)$ & $42.8( \pm 0.2)$ \\
\hline & $n$-Heptane & $24.15( \pm 0.4)$ & $12.64( \pm 0.2)$ & $15.25( \pm 0.1)$ \\
\hline \multirow[t]{5}{*}{$n$-Octane } & 3-Methyl heptanes & $18.6( \pm 0.8)$ & $21.4( \pm 0.8)$ & $13.2( \pm 0.1)$ \\
\hline & 3-Ethyl hexane & $22.8( \pm 1.1)$ & $27.3( \pm 1.1)$ & $21.2( \pm 0.3)$ \\
\hline & 3,3-Dimethyl hexane & $2.1( \pm 0.04)$ & $10.6( \pm 0.03)$ & $12.4( \pm 0.1)$ \\
\hline & 2,3-Dimethyl hexane & $2.7( \pm 0.1)$ & $16.9( \pm 0.03)$ & 11.7. $( \pm 0.8)$ \\
\hline & 2,3-Dimethyl-2-hexene & $0.29( \pm 0.05)$ & $0.33( \pm 0.02)$ & $0.42( \pm 0.05)$ \\
\hline
\end{tabular}

chloroaluminate ILs. ${ }^{47}$ There are several reasons for the observed increase in catalytic efficiency. Both catalysts contain Brønsted acid and Lewis acid sites, which catalyze the isomerization. In addition, incorporation of the silica matrix increases the surface area of catalyst. Importantly, the electronwithdrawing $\mathrm{HSO}_{4}{ }^{-}$group makes the $\mathrm{Si}^{4+}$ center a stronger Lewis acid. Finally, the large bisulfate group generates electronic polarization in the material, which enhances the proton conductivity of the catalyst. Thus, Brønsted acidity increases for CAT-1 and CAT-2. A possible mechanism for the formation of the desired branched-chain isomeric alkanes follows: a 'naked' proton is abstracted/released from $n$-heptane or $n$-octane, resulting in the formation of a carbocation, which subsequently undergoes rearrangement to produce the isomeric alkanes. Both catalysts have isomerized $n$-heptane and $n$-octane in high yields. From Tables 3 and 4, we estimate that CAT-1 is a more effective catalyst.

\section{Conclusions}

IL Brønsted acids 1-methyl imidazolium hydrogen sulphate ([HMIM] $] \mathrm{HSO}_{4}$ ) and 1-methyl benzimidazolium hydrogen sulphate ([HMBIM] $\mathrm{HSO}_{4}$ ) were synthesized and characterized by ${ }^{1} \mathrm{H}$ NMR, ${ }^{13} \mathrm{C}$ NMR, FT-IR, and mass spectrometry. Acidity and thermal stability of the ILs was evaluated using UV-Vis and TGA, respectively. ILs were prepared via a facile and inexpensive route that released no halogen by products. ILs were immobilized on silica gel to prepare silica-supported IL catalysts (SILC) [HMIM] $\mathrm{HSO}_{4} @$ silica and [HMBIM] $\mathrm{HSO}_{4} @$ silica. FT-IR, SEM, $\mathrm{N}_{2}$-adsorption-desorption (BET), $\mathrm{NH}_{3}$-TPD, and TGA were used to characterize the silica-supported IL catalysts. High activity and selectivity, ease of product separation, and recyclability make the immobilization of ILs catalysts advantageous. TGA data revealed that the benzene-substituted IL, [HMBIM] $\mathrm{HSO}_{4}$, had increased thermal stability compared to [HMIM] $\mathrm{HSO}_{4}$. Hence, [HMBIM] $\mathrm{HSO}_{4}$ is more stable. BET results demonstrated that the surface area of the silica-supported IL is smaller than that of pure silica. However, the attachment of bulky imidazolium or benzimidazolium cations to the framework contributes to higher strain on the mesostructure, which likely leads to increased catalytic activity. Both synthesized catalysts were used to isomerize $n$-heptane and $n$-octane at room temperature. Isomerization is more efficient with CAT- 1 than with CAT-2. The protocol described herein is suitable for environmentally friendly industrial applications. Efforts to immobilize ILs on silica and apply them for more complex olefin and paraffin isomerization reactions are ongoing in our group.

\section{Conflicts of interest}

No conflict of interest was stated by the authors.

\section{Acknowledgements}

The King Saud University authors would like to extend their sincere appreciation to the Deanship of Scientific Research at the King Saud University for its funding for this research group project No. (RG-1435-078).

\section{References}

1 J. Dupont, R. F. de Souza and P. A. Z. Suarez, Chem. Rev., 2002, 102, 366.

2 F. Jutz, J. M. Andanson and A. Baiker, Chem. Rev., 2011, 111, 322.

3 M. E. Zakrzewska, E. Bogel-Lukasik and R. Bogel-Lukasik, Chem. Rev., 2011, 111, 397.

4 M. Haumann and A. Riisager, Chem. Rev., 2008, 108, 1474.

5 M. Petkovic, K. R. Seddon, L. P. N. Rebelo and C. S. Pereira, Chem. Soc. Rev., 2011, 40, 1383.

6 K. Binnemans, Chem. Rev., 2007, 107, 2592.

7 F. van Rantwijk and R. A. Sheldon, Chem. Rev., 2007, 107, 2757.

8 T. L. Greaves and C. J. Drummond, Chem. Rev., 2008, 108, 206.

9 N. V. Plechkova and K. R. Seddon, Chem. Soc. Rev., 2008, 37, 123. 
10 D. Zhao, M. Wu, Y. Kou and E. Min, Catal. Today, 2002, 74, 157.

11 R. L. Vekariya, J. Mol. Liq., 2017, 227, 44.

12 T. Welton, Coord. Chem. Rev., 2004, 248, 2459.

13 M. J. Earle and K. R. Seddon, Pure Appl. Chem., 2000, 72, 1391.

14 R. D. Rogers and K. R. Seddon, Science, 2003, 302, 792.

15 K. Qiao and Y. Q. Deng, J. Mol. Catal. A: Chem., 2001, 171, 81.

16 C. Z. Qiao, Y. F. Zhang, J. C. Zhang and C. Y. Li, Appl. Catal., A, 2004, 276, 61.

17 V. A. Ksenofontov, T. V. Vasina, Y. E. Zubarev and L. M. Kustov, React. Kinet. Catal. Lett., 2003, 80, 329.

18 K. Herbst, J. Houzvicka, B. T. Jespersen, J. Zavilla, Inventors; HaldorTopsoe A/S, assignee, EP Patent 1346768, September 24, 2003.

19 R. Zhang, X. H. Meng, Z. C. Liu, J. Y. Meng and C. M. Xu, Ind. Eng. Chem. Res., 2008, 47, 8205.

20 Y. L. Gu, F. Shi and Y. Q. Deng, Catal. Commun., 2003, 4, 597.

21 S. Q. Yang, Z. C. Liu, X. H. Meng and C. M. Xu, Energy Fuels, 2009, 23, 70 .

22 C. Feher, E. Krivan, J. Hancsok and R. Skoda-Foldes, Green Chem., 2012, 14, 403.

23 G. A. Olah, US Pat., 3766286, 1973.

24 A. Dhar, N. S. Kumar, K. Sarkar, A. S. Al-Fatesh, A. A. Ibrahim, A. H. Fakeeha and R. L. Vekariya, J. Mol. Liq., 2019, 288, 111047.

25 W. Wang, C.-J. Liu and W. Wu, Catal. Sci. Technol., 2019, 9, 4162.

26 J. Chen, L. Yang, W. Zhou, L. Zhu, Y. Zhou, Y. Xiang and D. Xia, Energy Fuels, 2018, 32, 5518.

27 E. Rafiee, S. Rashidzadeh and A. Azad, J. Mol. Catal. A: Chem., 2007, 261, 49.

28 I. Mohammadpoor-Baltork, M. Moghadam, S. Tangestaninejad, V. Mirkhani and S. F. Hojati, Polyhedron, 2008, 27, 750.

29 A. B. Khan, L. H. Choudhury and S. Ghosh, J. Mol. Catal. A: Chem., 2006, 255, 230.
30 S. Dara, V. Saikam, M. Yadav, P. P. Singh and R. A. Vishwakarma, Carbohydr. Res., 2014, 391, 93.

31 A. R. Kiasat, M. Zayadi and M. F. Mehrjardi, Chin. Chem. Lett., 2008, 19, 665.

32 H. Eshghi and A. Hassankhani, J. Korean Chem. Soc., 2007, $\mathbf{5 1}, 361$.

33 K. Rajkumare and S. Rajendra, J. Mol. Catal. A: Chem., 2011, 345, 117.

34 R. L. Vekariya, A. Dhar and J. Lunagariya, Compos. Interfaces, 2017, 24, 801.

35 C. P. Fredlake, J. M. Crosthwaite, D. G. Hert, S. Aki and J. F. Brennecke, J. Chem. Eng. Data, 2004, 49, 4.

36 M. T. Clough, K. Geyer, P. A. Hunt, J. Mertes and T. Welton, Phys. Chem. Chem. Phys., 2013, 15, 20480.

37 C. Maton, N. D. Voss and C. V. Stevens, Chem. Soc. Rev., 2013, 42, 5963.

38 G. Liu, Z. L. Wang, M. J. Jia, X. J. Zou, X. M. Zhu, W. X. Zhang and D. Z. Jiang, J. Phys. Chem. B, 2006, 110, 16953.

39 Y. Jin, P. J. Wang, D. H. Yin, J. F. Liu, H. Y. Qiu and N. Y. Yu, Microporous Mesoporous Mater., 2008, 111, 569.

40 B. Karimi, D. Elhamifar, O. Yari, M. Khorasani, H. Vali, J. H. Clark and A. J. Hunt, Chem.-Eur. J., 2012, 18, 13520.

41 L. Wang, S. Shylesh, D. Dehe, T. Philippi, G. Dorr, A. Seifert, Z. Zhou, M. Hartmann, R. N. Taylor, M. J. Jia, S. Ernst and W. R. Thiel, ChemCatChem, 2012, 4, 395.

42 D. Liu, P. Yuan, H. M. Liu, J. G. Cai, Z. H. Qin, D. Y. Tan, Q. Zhou, H. P. He and J. X. Zhu, Appl. Clay Sci., 2011, 52, 358. 43 R. C. Ravindra, G. Nagendrappa and B. S. Jai Prakash, Catal. Commun., 2007, 8, 241.

44 A. Azzouz, D. Nistor, D. Miron, A. V. Ursu, T. Sajin, F. Monette, P. Niquette and R. Hausler, Thermochim. Acta, 2006, 449, 27.

45 O. V. Dzhikiya, M. D. Smolikov, K. V. Kazantsev, T. V. Kireeva and A. S. Belyi, Katal. Prom-sti., 2018, 18, 48.

46 A. A. Ibragimov, R. R. Shiriyazdanov, A. R. Davletshin and M. N. Rakhimov, Theor. Found. Chem. Eng., 2013, 47, 66.

47 C. Meyeraand and P. Wasserscheid, Chem. Commun., 2010, 46, 7625 . 